\title{
TMN/C++: An object-oriented API for GDMO, CMIS, and ASN.1
}

\section{T.R. Chatt}

Vertel

21300 Victory Blvd., Woodland Hills, CA 91367, USA

Phone: +1(818)227-1400 Fax: +1(818)598-0047

e-mail:tom-chatt@vertel.com

M. Curry, TCSI Corporation, mikec@tcs.com

J. Seppä, Nokia Telecommunications

juha.seppa@ntc.nokia.com

\section{U. Hollberg, IBM European Networking Center hollberg@heidelbg.ibm.com}

\begin{abstract}
The TMN/C++ API offers a standardized object-oriented application programming interface (API) for telecommunications management applications. The architecture comprises three modular, layered APIs. The ASN.1/C++ API provides a roleindependent interface to the data itself and its encoding. The CMIS/C++ API provides a service-oriented interface for the basic management information model (MIM) services - creating and deleting objects, getting and setting their attributesin an agent or manager role. The GDMO/C++ API provides a MIM-oriented framework for accessing and implementing managed objects in a containment tree and accessing CMIS services in an agent or manager role. The API set supports two distinct application types: "specific" applications which efficiently implement a static MIB specification, and "generic" applications which can dynamically interpret new MIBs. By the end of 1997, these standards documents are expected to be published as X/Open preliminary specifications and adopted by the NMF.
\end{abstract}

Keywords

TMN, GDMO, CMIS, ASN.1, C++, API, telecommunications, OSI, network management, agent, manager, MIM, NMF 


\section{PROJECT BACKGROUND}

By 1994, the notation, service model, and protocol to be used in telecom management software had been fairly well standardized by organizations such as the International Telecommunications Union (ITU, formerly the CCITT), and the Joint Technical Committee (JTC1) of the International Organization for Standardization (ISO) and the International Electrotechnical Commission (IEC). The Guidelines for Definition of Managed Objects (GDMO) established a notation and an objectoriented framework for describing management information. ${ }^{1}$ The Common Management Information Service (CMIS) specified the way in which such information would be exchanged across a network. ${ }^{2}$ And Abstract Syntax Notation One (ASN.1) standardized a machine-independent notation for representing data and a practical means of exchanging it. ${ }^{3}$ This level of "meta-standardization" provided a framework for standardizing information exchange for particular types of equipment and services. Once the framework was established, industry organizations such as the ITU-T and the Network Management Forum (NMF) could focus on standardizing the information required to manage equipment (such as SONET or SDH network elements) and enable services (such as electronic bonding). The combination of standard information and a standard means of communicating it enabled network interoperability between various manufacturers' equipment and the management software of various service providers and carriers.

With the adoption of the TMN model and the obvious advantages it offered came a need for a significant amount of complex software to implement it. Though the applications to be built on the TMN model may be quite heterogeneous, the commonality inherent in the standard information model and transport allowed the development of reuseable software for any TMN application. The software market responded by providing a variety of TMN software tools, generally comprising compilers which translated the standard GDMO and ASN.1 abstract notation into some specific programming language form, and "platforms" or "toolkits" which provided a CMIP engine in an "agent" or "manager" role. While these tools facilitated the development of TMN software applications, their application programming interfaces were non-standard and often complex. The learning curve invested in developing a TMN application using a particular vendor's TMN tools was often steep, and was not applicable to another vendor's TMN tools. Thus, application builders incurred repeated learning curve costs to use various tools as best fit particular applications, or else they were locked in to one vendor for a "one tool fits all" solution.

${ }^{1}$ GDMO is specified in ITU-T Rec. X.722 I ISO/IEC 10165-4.

${ }^{2}$ CMIS is specified in ITU-T Rec X.721 I ISO/IEC 10165-2.

${ }^{3}$ ASN.1 was standardized in 1990 as ITU-T Rec X.208 I ISO/IEC 8824. Significant extensions to the notation caused it to be re-standardized in 1994 as ITU-T Rec X.680, X.681, X.682, and X.683 I ISO/IEC 8824-1 through 8824-4. 
In response to this need to standardize an API for TMN applications, X/Open promulgated XMP and XOM, which provided a C language API for managing objects, and a method for mapping GDMO/ASN.1 onto the XMP/XOM domain. ${ }^{4}$ The wide acceptance of this standard, and its incorporation into many of the commercial TMN platforms demonstrated the need for such a standard. However, the utility of this standard in the TMN domain was hampered by its high degree of abstraction. It is generally perceived as difficult to use, and most TMN platforms which boast XMP/XOM compliance also offer more easy-to-use API layers which hide it. Moreover, XMP/XOM was a C API, while $\mathrm{C}++$ was gaining increasing acceptance as a more natural language for implementing object-oriented methodologies such as GDMO.'

This was the industry context in which the TMN/C++ API was conceived. At the Network Management Forum general meeting in autumn of 1994, a group of member companies initiated a collaborative effort to design a portable, easy-to-use, object-oriented application programming interface (API) for telecommunications management applications using the TMN standard model. The project, which got fully underway in 1995, was co-sponsored by the Network Management Forum (NMF) and X/Open, and enjoyed participation and support from a variety of organizations:

- telecom service providers and equipment manufacturers, such as Nokia, Motorola, Ericsson/HPT and Hitachi Telecom

- hardware platform vendors, including IBM, Sun, and HP

- $\quad$ TMN software platform providers, such as Vertel and TCSI

- $\quad$ software tool vendors, such as OSS, Opening Technologies, and DSET

- other industry and software standards organizations, such as the Open Software Foundation (OSF), and the International Organization for Standardization (ISO)

- other (non-telecom) ASN.1-based service providers, such as 3M Health Care.

The three-year project will produce a series of related standards documents to be adopted by the NMF and published by X/Open. The standards, which are coherent but loosely coupled, are being published separately as they become available and approved. The first two standards, TMN/C++ and ASN.1/C++, are technically approved and ready for publication at the time of this writing. It is anticipated that the entire series will be completed by the end of 1997 .

${ }^{4}$ See X/Open Documents C315 OSI - Abstract Data Manipulation API (XOM) and C306 Systems Management - Management Protocol API (XMP)

5 By early 1995, telecom companies including Nokia, NTT, and Ericsson/HPT, who had developed in-house TMN application platforms had done so using $\mathrm{C}++$. 


\section{DESIGN GOALS AND OVERALL ARCHITECTURE}

The working group agreed upon a set of goals for the API, with "simple, intuitive, and easy to use" being at the top of the list. The most natural expression of the object-oriented TMN model in a programming language was desired. Source code level portability was a second key goal, such that application developers would only have to invest in one learning curve for a common API and would not be locked in to particular vendors and tools. It was agreed that the complete functionality of GDMO, CMIS, and ASN.1 should be provided for, rather than trying to restrict the API to some subset of the model. The diversity of applications to be built upon the API-from network element agents which may be deployed on embedded processors to service management layer applications likely to be on large corporate information system platforms - demanded that the API be flexible and optimizable enough to meet these various needs. Finally, the commercial side of those present required that the API not be so grandiose as to be unimplementable in a reasonable market timeframe.

The $\mathrm{C}++$ programming language was chosen both for its natural strength in expressing an object-oriented model, and for its increasing deployment in the telecom industry and in the software industry in general. The use of $\mathrm{C}++$ class inheritance hierarchies to model ASN.1 and GDMO objects was adopted as the most intuitive way to translate these abstract notations into a programming language. Rather than limit the TMN/C++ API to the commercially available features of $\mathrm{C}++$ as of 1995 (which would have looked a bit stilted in a TMN/C++ standard published in 1997), the decision was taken to target the emerging ANSI standard for $\mathrm{C}++$ as a programming language baseline. ${ }^{6}$ All features were made use of, including virtual functions, templates, and exceptions. In particular, extensive use was made of the ANSI standard libraries, including strings and containers/iterators (the "standard template library" or STL).

The API was developed to accommodate two rather different software application models, which were termed the "specific" and the "generic" models. In the "specific" application model (see Figure 1), the application is designed around a particular set of management information which is established before development occurs. This information, fixed in a particular set of GDMO/ASN.1 definitions, is passed through a translation process (ie., a compiler) and specific $\mathrm{C}++$ classes are produced which model the information. In this model, a TMN/C++ API implementation would provide a base library and header files with $\mathrm{C}++$ classes representing the basic types and objects defined in GDMO and ASN.1, and the CMIS services. The API implementation would also provide a compiler which would take GDMO and ASN.1 inputs and generate specific classes for that particular information model. The application developer would then develop the application using these base classes, plus the compiler-generated classes (which are

${ }^{6}$ See ANSI X3J16/95-9987 (April 1995), Draft International Standard for Information Processing: Programming Language: $\mathrm{C}++$. 


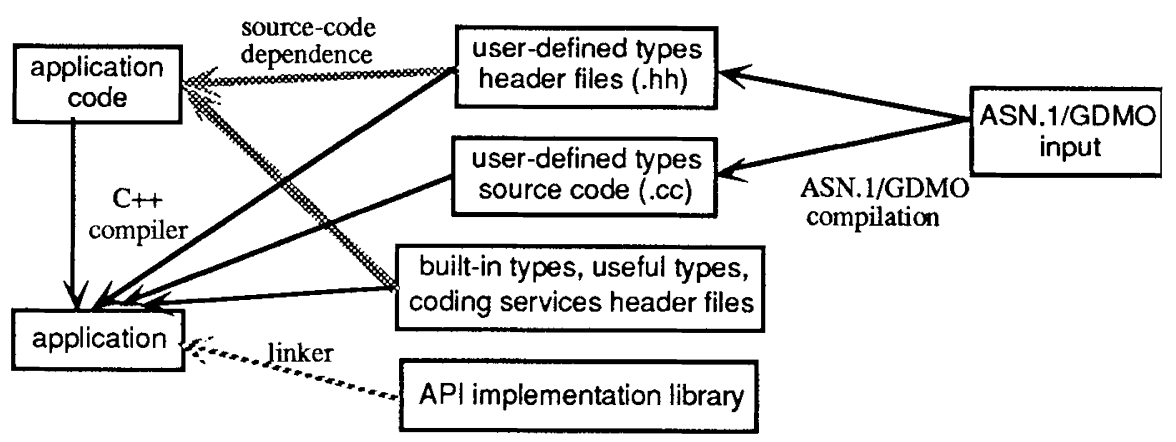

Figure 1 The "Specific" application model.

fixed at application development time). The specific model offers the benefits of a type-safe and easier-to-use interface by producing $\mathrm{C}++$ classes tailored for a specific application. This model is also likely to produce more efficient code in the final application.

In contrast, the "generic" model (see Figure 2) allows knowledge of the management information definition to be postponed until runtime. In this model, a TMN/C++ API implementation provides only a base library and header files defining $\mathrm{C}++$ classes representing the basic types and objects of GDMO and ASN.1, along with the ability to construct user-defined GDMO objects and ASN.1 types at runtime (eg., by loading and interpreting new or revised GDMO and ASN.1 specifications). The application developer would then develop an application using generic classes, and would construct management information definitions "on the fly". The generic model involves an exposed interface to the "meta-data", that is, the data which describes the structure of the management information. This model opens up the powerful possibility of an application which discovers new information definitions as it runs, and adapts to handle these new definitions. A generic application runs in an interpretive, rather than a compiled, mode.

Since the generic and specific application models differ, different APIs are needed to address them. Both the generic and specific APIs can stand alone, and TMN/C++ API implementations may decide to offer only one or the other. However, the

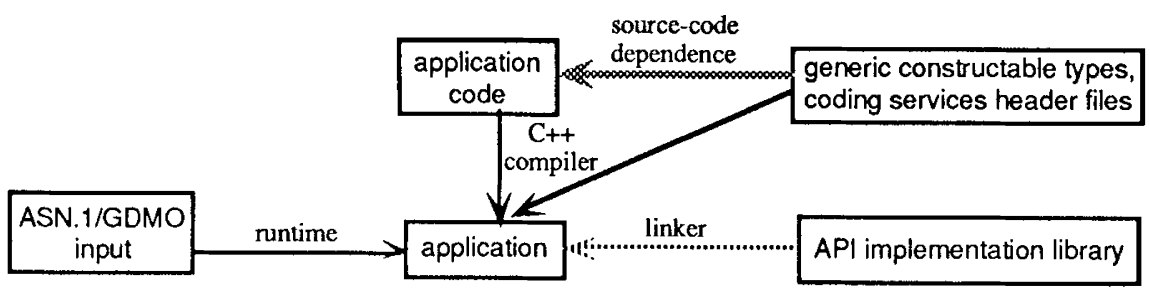

Figure 2 The "Generic" application model. 


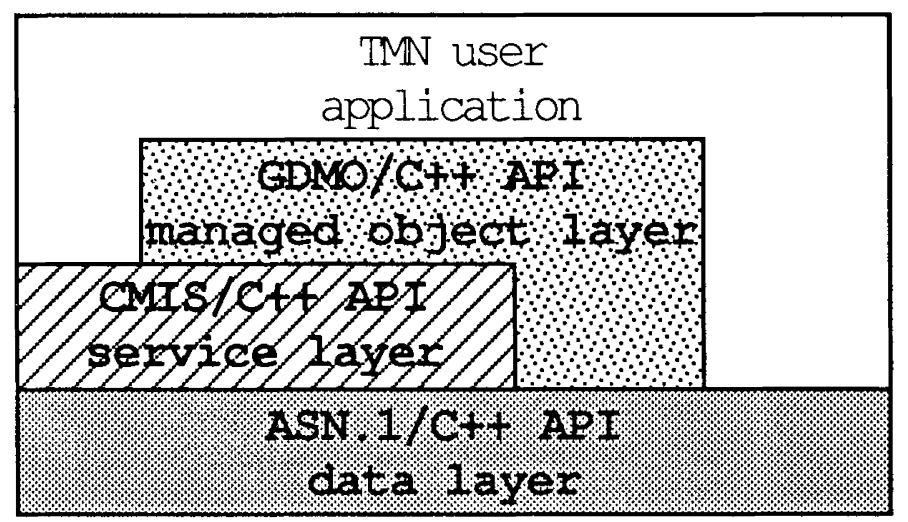

Figure 3 TMN/C++ layered architecture.

overall API was designed such that both generic and specific APIs together form one coherent API, and are not completely distinct. This allows one application to easily support a combination of compiled and dynamically interpreted information, and also simplifies both the understanding and the implementation of the complete TMN/C++ API.

The overall project of a TMN/C++ API seemed most naturally decomposed into three relatively independent, layered APIs. (See Figure 3.) The foundational layer is the interface to the management information (data) itself. Thus, the ASN.1/C++ API was conceived to provide a mapping from data syntax specifications (expressed in ASN.1) onto $\mathrm{C}++$ classes and objects for actual programmatic manipulation of the data. This layer deals strictly with data access and encoding, and is independent of the use of this data in the context of an X.700 management information model in an agent or manager role. Both "specific" and "generic" interfaces are offered in this layer, to allow for the efficient implementation of a static specification, and the flexible implementation of a dynamically interpretive application. Recognizing that ASN.1 has much wider applicability outside of TMN, the ASN.1/C++ API was designed to be a "stand alone" API, independent of the other two TMN/C++ API components.

The service layer, CMIS/C++ API, provides a programmatic interface to the services required to operate an agent or manager in a management information model (eg., creating and deleting objects, getting and setting their attributes). This layer provides the framework for an agent or manager "engine", and thus provides symmetric interfaces for the agent and manager roles (though the two roles may be coherently combined in one application). However, because this layer is independent of any particular management information base (MIB), it is by its nature only "generic", and has no "specific" components. The CMIS/C++ API is actually broader than just CMIS. It also includes the services defined in ACSE for initiating, responding to, and controlling associations between agents and managers. 
The managed object layer, GDMO/C++ API, provides a programmatic interface for navigating both inheritance trees (class definition meta-data) and containment trees (instances of management data). It also integrates the functionality of the service layer, representing it as methods associated with managed objects. This layer, which is the most closely tied to the X.700 model, has different (and not entirely symmetric) interfaces for agent and manager roles. (As with the service layer, agent and manager APIs may be coherently combined in one application.) And since this layer maps GDMO specifications onto $\mathrm{C}++$ classes and objects, it has both "specific" and "generic" variants.

\section{ASN.1/C++ API}

The ASN.1/C++ API defines a mapping from ASN.1 abstract entities, such as types and values, onto $\mathrm{C}++$ entities such as classes and objects. For each ASN.1 type, there is a corresponding C++ class.For each ASN.1 value, there is a corresponding $\mathrm{C}++$ object. ASN.1 modules, which encapsulate ASN.1 definitions, are translated as $\mathrm{C}++$ namespaces, which scope the corresponding classes and objects. The goal was to define a simple, easy-to-understand correspondence between the ASN.1 model and the C++ produced.

An abstract base class, Abst ractData, heads the $\mathrm{C}++$ class hierarchy which is used to model all ASN.1 types. (See Figure 4.) The AbstractData class provides all the functionality which is inherent in any item of ASN.1 data: it can be encoded and decoded, transformed to or from ASN.1 value notation, tested for validity against its subtype constraints, queried to discover its type information, and compared against other values. This class is abstract in the C++ sense (ie., contains pure virtual functions) and cannot be instantiated directly. Rather, it serves to provide a useful abstraction through which instances of its subclasses may be manipulated.

For each ASN.1 built-in type, a C++ subclass of AbstractData is defined to provide a type-specific "natural" representation. For instance, the $\mathrm{C}++$ class BOOLEAN, which represents the ASN.1 type BOOLEAN, derives from AbstractData, but otherwise has the look and feel of the $\mathrm{C}++$ bool built-in type. It is freely constructable from, assignable from, and castable to the $\mathrm{C}++$ bool type. Similarly, the C++ class INTEGER, which represents the ASN.1 type INTEGER, has the look and feel of a C++ int type.?

For user-defined ASN.1 types, the derivation of the corresponding $\mathrm{C}++$ classes follows the type definition. For instance, given an ASN.1 type 'Rate ::= INTEGER', the corresponding C++ class Rate derives from the $\mathrm{C}++$ class

7 This is actually a simplification. The actual specification provides for "huge" integers (ie., those which exceed the capacity of a given machine's native 'int'), as well as multiple INTEGER classes to make most efficient use of short and long ints. 


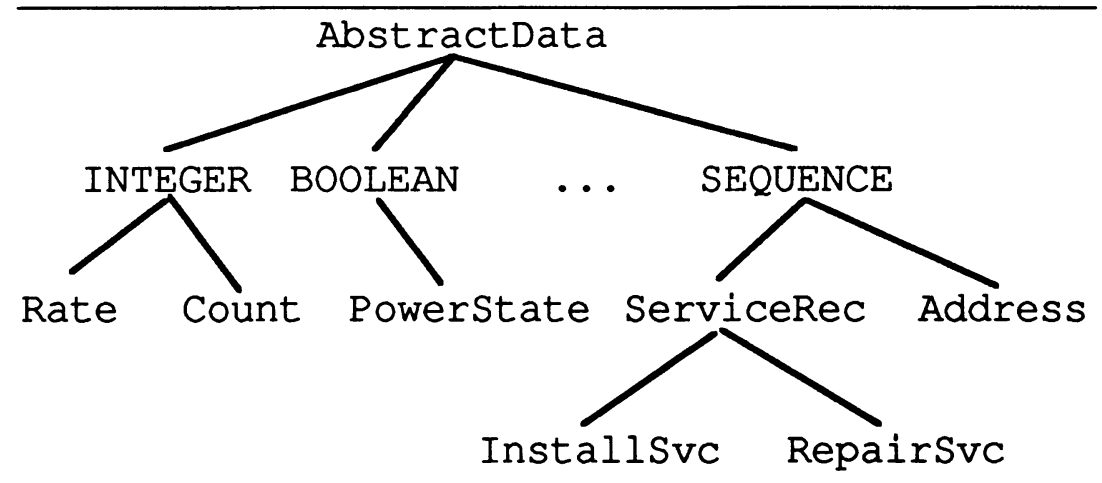

Figure 4 ASN.1 Base Class Hierarchy.

INTEGER. And given an ASN.1 type 'InstallSvc ::= ServiceRec', the corresponding $\mathrm{C}++$ class Installsvc would derive from the $\mathrm{C}++$ class ServiceRec.

The application interacts with the ASN.1/C++ API across four basic kinds of interface: abstract data, information objects, encoded buffers, and coding services. (See Figure 5.) The abstract data interface is through the AbstractData class hierarchy described above. In the specific ASN.1/C++ API, the interface includes type-safe generated $\mathrm{C}++$ classes to represent specific ASN.1 user-defined types. In the generic ASN.1/C++ API, the application manipulates data using the base classes and generic access methods (eg., to access components of structured types by field name or position). Similarly, the application may manipulate STL containers which represent ASN.1 information object sets, either through type-safe generated classes or generically.

The coding services are provided in the form of template functions for "encode" and "decode", which take an AbstractData subclass object as an argument. Following the style of an STL algorithm, the encode and decode functions may operate on any buffer which provides an octet (ie., unsigned char) iterator. This allows the implementation of buffers to be vastly open-ended. A buffer may

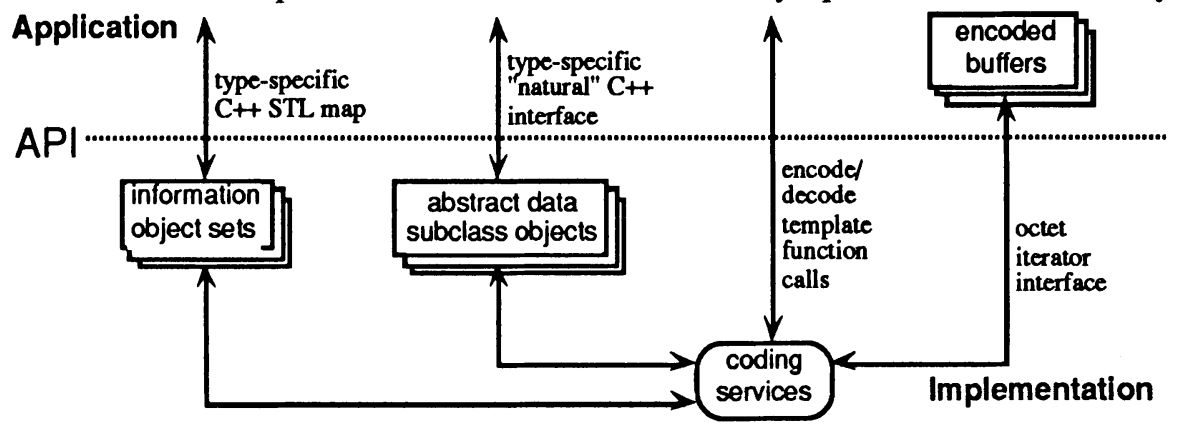

Figure 5 ASN.1 Application Interfaces. 
be as simple as an unsigned char*, or it may be any $\mathrm{C}++$ class which can "look" like one. An implementation may even provide "smart" buffer classes which can provide an efficient bypass around the one-octet-at-a-time public interface.

Where $\mathrm{C}++$ code is provided or generated to represent specifications expressed in ASN.1, the names of the C++ classes and objects are-to the greatest extent possible-made the same as the names of the ASN.1 entities they represent. Case is preserved and hyphens are mapped to underscores. Where limits on the length of names are needed, a standard algorithm for name shortening is provided. Rules are also provided for generating names where no names exist in the ASN.1 (anonymous fields and types), as well as for resolving keyword conflict and disambiguation issues.

The mapping of ASN.1 entities onto C++ causes ssome issues to arise, such as compatibility for assignment and comparison, which are not explicitly addressed in ASN.1 itself. The ASN.1/C++ class hierarchy implements a general rule of compatibility within any class inheritance subtree with a concrete common base class. For example, all INTEGER subtypes are compatible, and all subtypes of a particular SEQUENCE or SET type are compatible, but different SEQUENCEs and SETs are not compatible. This provides an easily understandable rule which is naturally coherent with the ASN.1/C++ class hierarchy.

For several reasons, including the convenient storage of ASN.1/C++ objects in STL containers, it was deemed useful to define comparison rules within and among all types. These comparison rules are semantically sensible where feasible (eg., string types are lexicographically ordered), and arbitrary where not.

The realization of the abstract notation into $\mathrm{C}++$ also introduced the need for directives in an ASN.1 specification, where supplemental information is needed or desireable to guide the translation process. Directives may control $\mathrm{C}++$ name generation, memory storage issues, and implementation variations. Thus, a standard directive format, which is a "distinguished" ASN.1 comment, was specified. The directive notation is position-independent and relocatable. By design, directives may enhance the translation process, but are never mandatory, so that "pure" ASN.1 is always translatable.

\section{CMIS/C++ API}

The CMIS/C++ API provides a programming interface to the OSI applicationservice-elements (ASEs) that are used by all management applications, namely the CMISE and the ACSE. The software components of the API are:

- a set of $\mathrm{C}++$ classes that model the application-service-elements, and their service primitives and parameters;

- objects for referring to outstanding operations (invocation handles), and mechanisms for applications to receive indication and confirmation service primitives (callback and queue classes);

- a "convenience" API for automatic association management. 
The basic idea behind the CMIS/C++ API design was to give the application developer a service-oriented (as opposed to protocol-oriented) view of the communications functions used by a management application. This was because a user's basis for understanding the communications functions provided by the ACSE and the CMISE are the service definition documents, not the protocol specifications. The service definitions document the meanings of the service interactions, their parameters, and the relationships between the parameters. These semantics are primarily what an application developer cares about.

Moreover, the service definitions use a very simple and consistent style for documenting service primitives and their parameters. It would have been possible to model the relatively more complex APDUs of the protocol definitions as $\mathrm{C}++$ objects (and indeed, as ASN.1/C++ objects), but mapping between service primitives and APDUs, while necessary, is the proper function of a protocol machine, not of application logic. The higher level of conceptual abstraction found in the service definitions made the service view the more appropriate basis for an API design. The mechanics of constructing or interpreting APDUs is left to the API implementation.

The basic design approach for the CMIS/C++ API was to use object oriented techniques to build explicit and detailed models of the CMISE and the ACSE, and their service primitives and service parameters, as $\mathrm{C}++$ objects. The main reason to model these service elements explicitly was so that the service definition documents, along with typical textbook explanations of the OSI application layer structure, could in large measure stand as primary sources of reference documentation on the structure and semantics of the API. It was felt that the closer the parallels between these explanations of the service elements and their $\mathrm{C}++$ embodiment in the API, the smaller the conceptual leap that a prospective user would need to take to understand how to use the API.

Another compelling reason for fine-grained modeling of all of an ASE's features was discovered during the design discussions for the ACSE interface. The initial thinking was to provide only a simplified interface for association establishment. As discussions progressed, however, it became apparent that to provide any less than the full set of capabilities offered by the ACSE definition would lead to a design that failed to support certain applications' requirements. Presenting the ACSE's capabilities only in some pre-packaged "convenience interface" form amounted to imposing a policy on the way in which those capabilities could be used. The convenience of such an interface would be apparent only in applications whose requirements accorded with that policy: a convenience interface is only convenient if it does what you need.

Conceptual simplifications which departed from a close modeling of the OSI service elements were avoided. For instance, since CMIS is inherently asyncronous, the $\mathrm{CMIS} / \mathrm{C}++$ offers only an asyncronous interface. (A syncronous interface is offered at the higher-level GDMO/C++ interface.) However, $\mathrm{C}++$ modeling techniques enabled the construction of user-friendly semantic-oriented classes. The service parameters are a good example. 
The CMIS and ACSE service definition documents are purposefully silent on the exact form of service-defined parameter values, concentrating instead on their semantics. This allowed some degree of abstraction in the design of the $\mathrm{C}++$ service parameter classes, in that there was no a priori requirement, from the service perspective, that the parameter types be literal ASN.1 data objects as represented in protocol. In the CMIS/C++ API, the interfaces presented by the parameter classes are defined primarily in terms of their semantics at the service interface, rather than their structures in protocol.

A simple example of how such an abstraction is modeled in the CMIS/C++ API is the Scope class. Part of the definition of this class is:

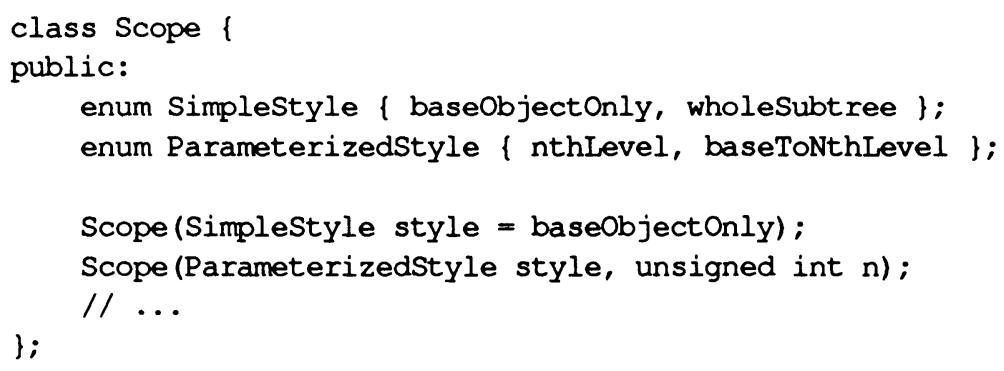

This abstraction aligns more intuitively with the semantics stated in the CMIS service definition document than does the ASN.1 type used to transmit the value (a CHOICE with three INTEGER alternatives).

Another powerful abstraction provided by the API is the use of $\mathrm{C}++$ operator overloading to simplify the construction of "filter" parameters using a symbolic paradigm. For example, an expression like:

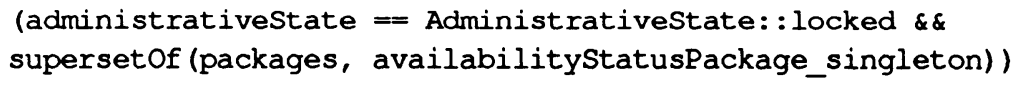

can be used to produce a filter parameter value with a fraction of the effort that would be required to construct the same value using only the ASN.1 types provided by the protocol specification.

\section{$5 \mathrm{GDMO} / \mathrm{C}++\mathrm{API}$}

The GDMO/C++ API provides programmers with a "managed object"-oriented API to CMIS services and to access and to implement managed objects. In the OSI management model, an agent role application contains managed objectsabstractions of managed resources-which it reveals to be managed by remote systems. A manager role application in turn issues management operations on managed objects contained within one or more agents. The GDMO/C++ API supports both manager and agent roles. Using the GDMO/C++ API, the user works with $\mathrm{C}++$ classes and objects which closely model the managed objects 
described in the GDMO for the MIB being implemented. This provides a natural realization of the OSI management model, where managed resources are modeled with managed objects.

The main goals of the GDMO/C++ API are:

- Provide an easy-to-use programming interface.

- Provide high level abstraction close to OSI management model.

- Hide as much of the complexities of communication as possible.

- Provide for strong compile time type checking when ever possible.

- Allow incremental development of management applications.

- Preserve all necessary information from GDMO specification.

The GDMO/C++ API provides a framework for management applications. This framework provides a context in which managed objects can be easily managed, by maintaining a context for each managed object instance, and automatically routing management operations to the appropriate managed object instance context. The framework validates all incoming and outgoing service requests, and provides default processing to handle all normal and error cases. In this manner, much of the complexity of communication is hidden.

Whereas the ASN.1/C++ API provides data types used in TMN, and the CMIS/C++ API provides services for communicating data, the GDMO/C++ API provides a complete application framework in which the application developer only need fill in the particular behavior of the managed objects being implemented. The API framework establishes a default implementation (usually returning a CMIS error) for all operations, which facilitates incremental application development. Programmers only need to define those parts important for the application, while everything else has been covered by API implementation. The framework thus enables faster and easier management application development.

Managed object classes defined in GDMO are modeled by "managed object handle" $(\mathrm{MOH}) \mathrm{C}++$ classes for manager role applications, and by "managed object" (MO) $\mathrm{C}++$ classes for agent applications. The $\mathrm{MOH} \mathrm{C++} \mathrm{classes} \mathrm{provide} \mathrm{a}$ communications interface for a manager role application, through which it can issue management operations on managed objects and receive responses to the operations. The MO C++ classes provide the basic structures for agent applications to implement managed objects.

The frameworks for manager role applications vs. agent role applications differ in several notable ways. The most important is the organization of the managed objects. On the agent side, the management information tree (MIT) model (also known as the "containment tree") is closely followed, with each managed object represented by one $\mathrm{MO}$ instance in the tree. The agent role framework provides functionality to implement and manage this tree, including default automatic processing of scope and filter.

On the manager side, instead of imposing a tree structure, the notion of $\mathrm{MOH}$ collections is introduced. There are enumerated collections, in which the user may insert or delete any arbitrary MOH. There are also rule-based collections, which are 
constructed based on the result of scope-and-filter operations. The manager role user may arrange any number of $\mathrm{MOHs}$ and $\mathrm{MOH}$ collections as suits the application. On the manager side, multiple $\mathrm{MOHs}$ may provide an interface to the same managed object.

Like the ASN.1/C++ API, two different software models, generic and specific, need to be addressed by this API. The specific API is based on a fixed set of managed objects known at compile time, as opposed to the generic API which is able to handle new GDMO and ASN.1 definitions dynamically. In the specific model, each GDMO managed object class is translated or "mapped" onto a specific $\mathrm{C}++$ class which represents it. The specific $\mathrm{C}++$ classes provide type-safe interfaces based on the GDMO and ASN.1 definitions, and thus can provide compile-time type checking. In the generic model, a base $\mathrm{C}++$ managed object class is used to represent an instance of any GDMO managed object class. Each instance, however, is dynamically linked to type information for its particular managed object class. In this way the validity of any operation issued on a generic managed object instance can be checked at runtime.

Both manager and agent role frameworks offer an application shell with complete default behaviour which the user can specialize and override as necessary. Two basic mechanisms for this specialization are offered. A callback mechanism, essential for the generic model, allows the developer to register callbacks for particular events at various contexts (eg., an object instance, an object class, the framework). A C++ virtual function mechanism allows the developer to derive specialized classes from the mapped (or base) managed object classes, and to provide specialized implementations of the virtual methods. The latter method is more suited to the specific model, and works in concert with an "object factory", which is used by the framework to automatically instantiate objects when needed. The factory also uses a virtual function mechanism which allows the framework to instantiate user-defined classes.

The framework offers further interface simplifications. OSI management operations are inherently asynchronous. An operation consists of four phasesrequest, indication, response and confirmation-which CMIS models as service messages. The GDMO/C++ API provides management operations in two modes: asynchronous and synchronous. In the synchronous mode of operation the four phased operation is abstracted to a blocking function call. This model makes developing simple management applications easy, and is also useful for multithreaded applications. Not all applications can accommodate to synchronous mode (eg., single-threaded user interface applications, which need to perform other activities while a communications operation is being processed), and thus asynchronous mode is provided as well.

The framework also simplifies application development by providing a cache for selected managed object attribute values. On the manager side, the cache feature can be combined with an automatic tracking feature, which allows the manager application to treat its $\mathrm{MOHs}$ as $\mathrm{C}++$ objects which are remotely implemented, offering a highly simplified CORBA-like interface. 


\section{SUMMARY}

By exploiting the object-oriented modeling power of the C++ language, the TMN/C++ project has created an ensemble of highly modular APIs which greatly simplify the development of TMN management applications. By structuring the project as coherent but loosely-coupled APIs, a wide variety of different application domains can be suited. Lower-level message-oriented applications can have the greatest degree of control over their network interface by using $\mathrm{ASN} .1 / \mathrm{C}++$ by itself. Applications which want explicit control at the CMIS service and association control level will find that $\mathrm{CMIS} / \mathrm{C}++$ offers an easy-to-use embodiment of the service model. And ultimately, applications which want to operate at a high level of abstraction will find that GDMO/C++ offers a good combination of simplicity, flexibility, and power. Simplicity is achieved at each level by close C++ modeling of the semantics of ASN.1, CMIS, and GDMO and MIM, combined with leveraging of established $\mathrm{C}++$ technology such as the Standard Template Library. Common design idioms are deployed across all the APIs to achieve a consistent and coherent ensemble, furthering ease of use.

Several NMF member companies have already begun to prototype and even productize components of this emerging standard. Early reports indicate that it is commercially feasible to develop, and that users of the APIs find their application development significantly expedited.

\section{REFERENCES}

ANSI Working Paper X3J16/95-9987 (April 1995), "Draft Proposed International Standard for Information Processing: Programming Language: C++".

ITU-T Recommendation X.680 "Information Technology - Abstract Syntax Notation One (ASN.1): Specification of basic notation." (1994), also ISO/IEC 8824-1 (1995).

ITU-T Recommendation X.721 "Information Technology - Open Systems Interconnection - Common management information service definition" (1991), also ISO/IEC 9595.

ITU-T Recommendation X.722 "Information technology - Open systems interconnection - Structure of management information - Guidelines for the Definition of Managed Objects" (1992), also ISO/IEC 10165-4.

Network Management Forum.

TMN/C++ Application Programming Interface. NMF 039.

ASN.1/C++ Application Programming Interface. NMF 040.

CMIS/C++ Application Programming Interface. NMF 041.

GDMO/C++ Application Programming Interface. NMF 042. 


\section{BIOGRAPHY}

Tom Chatt is a Principal Software Engineer at Vertel in Woodland Hills, CA, with architectural responsibility for Vertel's TMN Manager and Agent Development Environment products. Michael A. Curry is a Principal Engineer for the TCSI Corporation in Berkeley, CA. Ulf Hollberg is a Senior Consultant at the IBM European Networking Center in Heidelberg, with architecural responsibility for IBM's TMN product components. Juha Seppä is a Project Manager at Nokia in Tampere. He has several years background in practical TMN implementations for GSM networks.

All of the authors have been active participants in the NMF High-Level API Working Group which is developing these standards. 